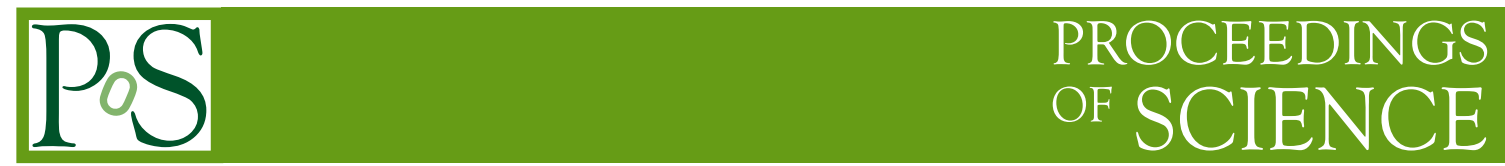

\title{
An improved model of color confinement
}

\section{Daniel Zwanziger*}

Physics Department, New York University, New York, NY 10003, USA

E-mail: dz2@nyu. edu

We consider the free energy $W[J]=W_{k}(H)$ of QCD coupled to an external source $J_{\mu}^{b}(x)=$ $H_{\mu}^{b} \cos (k \cdot x)$, where $H_{\mu}^{b}$ is, by analogy with spin models, an external "magnetic" field with a color index that is modulated by a plane wave. We report an optimal bound on $W_{k}(H)$ and an exact asymptotic expression for $W_{k}(H)$ at large $H$. They imply confinement of color in the sense that the free energy per unit volume $W_{k}(H) / V$ and the average magnetization $m(k, H)=\frac{1}{V} \frac{\partial W_{k}(H)}{\partial H}$ vanish in the limit of constant external field $k \rightarrow 0$. Recent lattice data indicate a gluon propagator $D(k)$ which is non-zero, $D(0) \neq 0$, at $k=0$. This would imply a non-analyticity in $W_{k}(H)$ at $k=0$.

We present a model that is consistent with the new results and exhibits (non)-analytic behavior. Direct numerical tests of the bounds are proposed.

The many faces of $Q C D$

November 2-5, 2010

Gent Belgium

${ }^{*}$ Speaker. 


\section{Introduction}

Recent numerical studies on large lattices of the gluon propagator $D(k)$ in Landau gauge in 3 and 4 Euclidean dimensions, reviewed recently in [1], yield finite values for $D(0) \neq 0$ [2] - [7], in apparent disagreement with the theoretical expectation that $D(0)=0$, originally obtained by Gribov [8], and argued in [9]. The argument [9] which leads to $D(0)=0$, relies on the hypothesis that the free energy $W(J)$ in the presence of sources $J$ is analytic in $J$ at low momentum $k$. That hypothesis should perhaps be dropped in view of the apparent disagreement with the lattice data. This is of interest because a non-analyticity in the free energy is characteristic of a change of phase.

The free energy $W(J)$ enters the picture because it is the generating functional of the connected gluon correlators. In particular the gluon propagator is a second derivative of $W(J)$ at $J=0$,

$$
D_{\mu v}^{a b}(x, y)=\left.\frac{\delta^{2} W(J)}{\delta J_{\mu}^{a}(x) \delta J_{v}^{b}(y)}\right|_{J=0} .
$$

The free energy $W(J)$ in the presence of sources $J$ is given by

$$
\begin{aligned}
\exp W(J) & =\langle\exp (J, A)\rangle \\
& =\int_{\Omega} d A \rho(A) \exp (J, A),
\end{aligned}
$$

where $\mu, v$ are Lorentz indices, and $a, b$ are color indices, and

$$
(J, A)=\int d^{D} x J_{\mu}^{b}(x) A_{\mu}^{b}(x) .
$$

The integral over $A$ is effected in Landau gauge $\partial_{\mu} A_{\mu}=0$, and the domain of integration is restricted to the Gribov region $\Omega$, a region in $A$-space where the Faddeev-Popov operator is nonnegative, $M(A) \equiv-\partial_{\mu} D_{\mu}(A) \geq 0$. We use continuum notation and results, but we have in mind the limit of lattice QCD in the scaling region, that is gauge-fixed to the Landau (or Coulomb) gauge by a numerical algorithm that minimizes the Hilbert norm squared $\|A\|^{2}$, and thereby fixes the gauge to the interior of the Gribov region. The vector potential, given by $A(x)=g A^{\text {pert }}(x)$, is unrenormalized, and has engineering dimension in mass units $[A(x)]=1$ in all Euclidean dimension $D$, while $[H]=D-1$. (Our results also hold in the Coulomb gauge at fixed time, in which case $D$ is the number of space dimensions.) The density $\rho(A)$ is a positive, normalized probability distribution with support in the Gribov region $\Omega$. Because there are Gribov copies inside $\Omega, \rho(A)$ is not unique and, in general, depends on the minimization algorithm.

We consider a source that has the particular form

$$
J_{\mu}^{b}(x)=H_{\mu}^{b} \cos \left(k x_{1}\right),
$$

where we have aligned the 1-axis along $k$, so the free energy

$$
\exp W_{k}(H)=\left\langle\exp \left[\int d^{D} x H_{\mu}^{b} \cos \left(k x_{1}\right) A_{\mu}^{b}(x)\right]\right\rangle,
$$

depends only on the parameters $k$ and $H_{\mu}^{b}$. This is sufficient to generate the gluon propagator for momentum $k$,

$$
D_{i j}^{a b}(k)=2 \frac{\partial^{2} w_{k}(0)}{\partial H_{i}^{a} \partial H_{j}^{b}}
$$


where

$$
w_{k}(H) \equiv \frac{W_{k}(H)}{V}
$$

is the free energy per unit Euclidean volume. Because $A_{\mu}(x)$ is transverse, only the transverse part of $H$ is operative, and we impose $k_{\mu} H_{\mu}^{b}=0$, which yields $H_{1}^{a}=0$, and we write $H_{i}^{a}$, where $i=2, \ldots D$. By analogy with spin models, $H_{i}^{b}$ may be interpreted as the strength of an external "magnetic" field, with a color index $b$, which is modulated by a plane wave $\cos \left(k x_{1}\right)$. (This external magnetic field $H_{i}^{b}$, with color index $b$, should not be confused with the Yang-Mills color-magnetic field $F_{i j}^{b}$.)

A rigorous bound for $W_{k}(H)$ on a finite lattice was given in [9] which holds for any (numerical) gauge fixing with support inside the Gribov region $\Omega$. One can easily show that in the limit of large lattice volume $V$, and in the continuum limit, this implies the Lorentz-invariant continuum bound in $D$ Euclidean dimensions,

$$
w_{k}(H) \leq\left(2 D k^{2}\right)^{1 / 2}|H|
$$

where $|H|^{2}=\sum_{\mu, b}\left(H_{\mu}^{b}\right)^{2}$. A model satisfying the bound (1.8) was recently exhibited in [10].

More recently, a stricter bound for $w_{k}(H)$ at finite $H$ was obtained [11], that also holds for any (numerical) gauge fixing with support inside the Gribov region $\Omega$,

$$
w_{k}(H) \leq 2^{-1 / 2} k \operatorname{tr}\left[\left(H^{a} H^{a}\right)^{1 / 2}\right] .
$$

Here $H^{a} H^{a}$ is the matrix with elements $H_{i}^{a} H_{j}^{a}$. It has positive eigenvalues, and the positive square root is understood. A proof may be found in the Appendix that this bound is stricter than the old bound (1.8). This bound is in fact optimal for a probability distribution $\rho(A)$ of which it is known only that its support lies inside the Gribov region.

The same expression also provides the asymptotic form of $w_{k}(H)$ at large $H$, and infinite Euclidean volume $V$ [11] for any numerical gauge fixing with probability density $\rho(A)$ with support that reaches all boundary points of $\Omega$, (but which may vanish on the boundary, $\rho(A)=0$ for $A \in \partial \Omega$ )

$$
w_{k, \text { as }}(H)=2^{-1 / 2} k \operatorname{tr}\left[\left(H^{a} H^{a}\right)^{1 / 2}\right] .
$$

Either bound yields in the zero-momentum limit

$$
w_{0}(H)=\lim _{k \rightarrow 0} w_{k}(H)=0
$$

As discussed in [9], this states that the system does not respond to a constant external colormagnetic field no matter how strong. It is a consequence of the proximity of the Gribov horizon in infrared directions. We shall return in the concluding section to the physical implications of this result for confinement of color.

If $w_{k}(H)$ were analytic in $H$ in the limit $k \rightarrow 0$, eq. (1.11) would imply that all derivatives of the generating function $w_{0}(H)$ vanish, including in particular the gluon propagator (1.6) at $k=0$, $D(0)=0$. However, as noted above, this disagrees with recent lattice data which indicate a finite value, $D(0) \neq 0$, in Euclidean dimensions 3 and 4. If this is true, then $w_{k}(H)$ must become nonanalytic in $H$ in the limit $k \rightarrow 0$. In order to get some insight about this, we examine the behavior of an improved model that has the exact asymptotic behavior (1.10). 


\section{Improved model}

The model is defined by the expression for the free energy

$$
w_{k, \bmod }(H)=g(k)\left\{\operatorname{tr}\left[\left(I+\frac{k^{2} H^{a} H^{a}}{2 g^{2}(k)}\right)^{1 / 2}-I\right]-\operatorname{tr} \ln \left[2^{-1}\left(I+\frac{k^{2} H^{a} H^{a}}{2 g^{2}(k)}\right)^{1 / 2}+2^{-1} I\right]\right\},
$$

where $g(k) \geq 0$ is an as yet undetermined function, and $H^{a} H^{a}$ is the matrix with elements $H_{i}^{a} H_{j}^{a}$, for $i, j=2, \ldots D$. This model possesses the following desirable features [11]: (i) It satisfies $w_{k, \bmod }(0)=$ 0 , which is correct at $H=0$ for a normalized probability distribution $\int d A \rho(A)=1$. (ii) It has the asymptotic limit

$$
w_{k, \text { as }}(H)=\lim _{\mu \rightarrow \infty} \frac{w_{k, \bmod }(\mu H)}{\mu}=2^{-1 / 2} k \operatorname{tr}\left[\left(H^{a} H^{a}\right)^{1 / 2}\right],
$$

that is correct at large $H$ for any numerical gauge fixing that is strictly positive in the interior of the Gribov region $\Omega$. (iii) It satisfies the optimal bound

$$
w_{k, \bmod }(H) \leq 2^{-1 / 2} k \operatorname{tr}\left[\left(H^{a} H^{a}\right)^{1 / 2}\right],
$$

which implies that the generating function vanishes at $k=0, w_{0, \bmod }(H)=0$. (iv) The matrix of second derivatives is positive in the sense that

$$
v_{i}^{a} \frac{\partial^{2} w_{k, \bmod }(H)}{\partial H_{i}^{a} \partial H_{j}^{b}} v_{j}^{b} \geq 0
$$

holds for all $v_{i}^{a}$ and $H_{i}^{a}$, as required for this matrix to be a covariance.

Because of the property $w_{0, \text { mod }}(H)=0$, there must be some non-analyticity if, as indicated by numerical calculations, the gluon propagator $D(k)$ at $k=0$ is positive $D(0)>0$. It is instructive to see what kind of analyticity this would be in our model. Let $\lambda(H)>0$ be the largest eigenvalue of the matrix $H_{i}^{a} H_{j}^{a}$. Inspection of (2.1) shows that $w_{k, \bmod }(H)$ is analytic in $H$ inside a radius of convergence

$$
\lambda(H)=\frac{2 g^{2}(k)}{k^{2}} .
$$

Moreover from (2.1) we have at small $H$,

$$
w_{k, \bmod }(H)=\frac{k^{2}}{8 g(k)} H_{i}^{a} H_{i}^{a}+g(k) O\left[k^{4} H^{4} / g^{4}(k)\right] .
$$

For the gluon propagator $D(k) \sim \frac{\partial^{2} w_{k \text { mod }}(0)}{\partial H_{i}^{a} \partial H_{j}^{b}} \sim k^{2} / g(k)$ to be finite at $k=0$, as suggested by the lattice data, we must have $g(k)=$ const $k^{2}$ near $k=0$. In this case the coefficient of the $H^{4}$ term is of order $1 / k^{2}$, which diverges as $k \rightarrow 0$, as do all higher order coefficients. Moreover the radius of convergence of the series expansion of $w_{k, \bmod }(H)$ is $\lambda(H)=O\left(k^{2}\right)$, which vanishes like $k^{2}$.

Suppose that $g(k)$ has a power law behavior $g(k) \sim k^{v}$ at $k=0$. Then the radius of convergence behaves like $\lambda(H) \sim k^{2 v-2}$, which approaches 0 with $k$ for $v>1$. The gluon propagator behaves like $D(k) \sim k^{2-v}$, and $w_{k \text {,mod }}(H)$ is non-analytic in $H$ at $k=0$ when the propagator has a power law $D(k) \sim k^{p}$ with $p<1$. Gribov's original calculation gave $D(k) \sim k^{2} / m^{4}$ which corresponds to $g(k)=O\left(m^{4}\right)$, and $w_{k, \bmod }(H)$ is analytic in $H$ at $k=0$, with a radius of convergence $\lambda(H)=$ $O\left(k^{-2}\right) \rightarrow \infty$ for $k \rightarrow 0$. 


\section{Conclusion}

By analogy with spin models, we define, for each momentum $k$, the analog of the bulk magnetization in the presence of the external "magnetic" field $H_{i}^{a}$,

$$
M_{i}^{a}(k, H)=\frac{\partial W_{k}(H)}{\partial H_{i}^{a}},
$$

which describes the reaction of the spin system to the external color-magnetic field. Its physical meaning in gauge theory is apparent from (1.5) which yields,

$$
\begin{aligned}
M_{\mu}^{b}(k, H) & =\left\langle\int d^{D} x \cos \left(k x_{1}\right) A_{i}^{b}(x)\right\rangle_{H} \\
& =(1 / 2)\left\langle a_{i}^{b}(k)+a_{i}^{b}(-k)\right\rangle_{H} .
\end{aligned}
$$

Thus the "bulk magnetization" is in fact the $k$-th fourier component of the gauge field in the presence of the external magnetic field. We also define the magnetization per unit (Euclidean) volume

$$
m_{i}^{a}(k, H)=\frac{M_{i}^{a}(k, H)}{V},
$$

given by

$$
m_{i}^{a}(k, H)=\frac{\partial w_{k}(H)}{\partial H_{i}^{a}} .
$$

The asymptotic free energy (1.10) determines the asymptotic magnetization per unit volume at large $H$,

$$
\begin{aligned}
m_{i, \mathrm{as}}^{a}(k, H) & =\frac{\partial w_{k, \mathrm{as}}(H)}{\partial H_{i}^{a}} \\
& =2^{-1 / 2} k\left[\left(H^{b} H^{b}\right)^{-1 / 2}\right]_{i j} H_{j}^{a} .
\end{aligned}
$$

Its magnitude is given by $\left(m_{i, \text { as }}^{a} m_{i, \text { as }}^{a}\right)(k, H)=k^{2} / 2$, and we obtain the simple formula

$$
\lim _{H \rightarrow \infty}\left(m_{i}^{a} m_{i}^{a}\right)(k, H)=k^{2} / 2,
$$

which holds for any numerical gauge fixing with support extending up to the boundary of the Gribov region $\Omega$.

We arrive at the remarkable conclusion that in the limit of constant external magnetic field, $k \rightarrow$ 0 , the color magnetization per unit volume vanishes, no matter how strong the external magnetic field,

$$
\lim _{k \rightarrow 0} \lim _{H \rightarrow \infty} m_{i}^{a}(k, H)=0 .
$$

Thus the system does not respond to a constant external color-magnetic field. In this precise sense the color degree of freedom $m_{i}^{b}(k, H)=\frac{1}{2 V}\left\langle a_{i}^{b}(k)+a_{i}^{b}(-k)\right\rangle_{H}$ is absent at $k=0$. This conclusion holds whether or not the free energy $w_{k}(H)$ is analytic in $H$ in the limit $k \rightarrow 0$. Lattice data would indicate that it is not analytic. Besides reporting this result, we have presented a model, defined in (2.1), which saturates the asymptotic limit (1.10), and exhibits confinement of color. As we have seen, $W_{k, \bmod }(H)$ may be either analytic in $H$, or not, at $k=0$, depending on the behavior of $g(k)$ 
at $k=0$, but in either case, the conclusion stands, that the constant color degree of freedom of the gauge field is confined.

Equations (1.9) and (1.10) may be checked numerically, at least in principle, by using the formula $\exp W_{k}(H)=\left\langle\exp \left[\int d^{D} x H_{i}^{b} \cos \left(k x_{1}\right) A_{i}^{b}(x)\right]\right\rangle$ to make a numerical determination of the generating function itself. For large values of $H$ this may fluctuate too wildly. Alternatively one may measure the magnetization from the formula $M_{\mu}^{b}(k, H)=\left\langle\int d^{D} x \cos \left(k x_{1}\right) A_{i}^{b}(x)\right\rangle_{H}$, where the source term $H_{i}^{b} \cos \left(k x_{1}\right) A_{i}^{b}(x)$ is included in the action that one simulates. This requires simulating the theory fixed in the Landau gauge instead of generating an ensemble from the gauge-invariant Wilson action then gauge fixing. It may be convenient to do this by numerical simulation of stochastic quantization [13] because that avoids calculating the Faddeev-Popov determinant explicitly.

\section{Acknowledgements}

The author recalls with pleasure stimulating conversations with Attilio Cucchieri and Axel Maas that took place at the conference, The Many Faces of QCD, November 2-5, 2010, Gent, Belgium. I am grateful to the conference organizers for the valuable opportunity which this conference provided.

\section{A. The new bound is stricter than the old}

To prove that the new bound is stricter we write the old bound (1.8), for the case that $k$ is aligned along the 1 -axis, as $w_{k}(H) \leq B_{1}$, where

$$
B_{1} \equiv(2 D)^{1 / 2} k\left[\operatorname{tr}\left(H^{a} H^{a}\right)\right]^{1 / 2} .
$$

We now diagonalize the matrix $H_{i}^{a} H_{j}^{a}$ by a rotation in the $(D-1)$-dimensional space, with eigenvalues $\lambda_{i}$, so

$$
B_{1}=(2 D)^{1 / 2} k\left(\sum_{i=2}^{D} \lambda_{i}\right)^{1 / 2}
$$

The new bound (1.9) reads $w_{k}(H) \leq B_{2}$ where

$$
B_{2}=2^{-1 / 2} k \sum_{i=2}^{D} \lambda_{i}^{1 / 2}
$$

and we must show $B_{2}<B_{1}$. (Note that the operations of square root and trace are interchanged.) We have

$$
B_{1}^{2}=2 D k^{2} \sum_{i=2}^{D} \lambda_{i}
$$

and

$$
\begin{aligned}
B_{2}^{2} & =2^{-1} k^{2} \sum_{i, j=2}^{D} \lambda_{i}^{1 / 2} \lambda_{j}^{1 / 2} \\
& =2^{-1} k^{2}\left(\sum_{i=2}^{D} \lambda_{i}+\sum_{i \neq j=2}^{D} \lambda_{i}^{1 / 2} \lambda_{j}^{1 / 2}\right)
\end{aligned}
$$




$$
\begin{aligned}
& \leq 2^{-1} k^{2}\left(\sum_{i=2}^{D} \lambda_{i}+2^{-1} \sum_{i \neq j=2}^{D}\left(\lambda_{i}+\lambda_{j}\right)\right) \\
& =2^{-2} k^{2} \sum_{i, j=2}^{D}\left(\lambda_{i}+\lambda_{j}\right) \\
& =2^{-1} k^{2}(D-1) \sum_{i=2}^{D} \lambda_{i} \\
& =(4 D)^{-1}(D-1) B_{1}^{2} .
\end{aligned}
$$

We thus obtain $B_{2}^{2} \leq(4 D)^{-1}(D-1) B_{1}^{2}<B_{1}^{2}$, so the new bound is stricter, as asserted.

\section{References}

[1] Attilio Cucchieri, Tereza Mendes arXiv:1001.2584 [hep-lat].

[2] Attilio Cucchieri, Tereza Mendes, arXiv:0812.3261 [hep-lat].

[3] Attilio Cucchieri, Tereza Mendes, Phys. Rev. D78 (2008) 094503 and arXiv: 0804.2371 [hep-lat].

[4] I.L. Bogolubsky, E.-M. Ilgenfritz, M. M§ller-Preussker, A. Sternbeck, arXiv:0901.0736 [hep-lat].

[5] O. Oliveira, P. J. Silva, arXiv:0809.0258 [hep-lat].

[6] A. Sternbeck, L. von Smekal, D. B. Leinweber, A. G. Williams PoS LAT2007 340, 2007 arXiv:0710.1982 [hep-lat].

[7] A. Cucchieri, T. Mendes, Phys. Rev Lett. 100 241601, 2008 and arXiv:0712.3517 [hep-lat].

[8] V. N. Gribov, Nucl. Phys. B 139, 1 (1978).

[9] Daniel Zwanziger Nucl. Phys. B 364, 127 (1991).

[10] Daniel Zwanziger arXiv:1012.2859 [hep-ph].

[11] Daniel Zwanziger (in preparation).

[12] A. Maas, Phys. Rev. D75 116004 (2007) and arXiv:0704.0722 [hep-lat].

[13] A. Nakamura and M. Mizutani, Vistas in Astronomy (Pergamon Press), vol. 37, 305 (1993); A. Nakamura, I. Pushkina, T. Saito and S. Sakai, Phys. Lett. B 594, 133 (2002). 